\title{
Article
}

Subscriber access provided by King Abdullah University of Science and Technology Library

\section{Revisiting nitrogen species in covalent triazine frameworks}

Dmitrii Yu. Osadchii, Alma Itzel Olivos Suarez, Anastasiya V. Bavykina, and Jorge Gascon

Langmuir, Just Accepted Manuscript • DOI: 10.1021/acs.langmuir.7b02929 • Publication Date (Web): 28 Nov 2017

Downloaded from http://pubs.acs.org on December 4, 2017

\section{Just Accepted}

"Just Accepted" manuscripts have been peer-reviewed and accepted for publication. They are posted online prior to technical editing, formatting for publication and author proofing. The American Chemical Society provides "Just Accepted" as a free service to the research community to expedite the dissemination of scientific material as soon as possible after acceptance. "Just Accepted" manuscripts appear in full in PDF format accompanied by an HTML abstract. "Just Accepted" manuscripts have been fully peer reviewed, but should not be considered the official version of record. They are accessible to all readers and citable by the Digital Object Identifier (DOI®). "Just Accepted" is an optional service offered to authors. Therefore, the "Just Accepted" Web site may not include all articles that will be published in the journal. After a manuscript is technically edited and formatted, it will be removed from the "Just Accepted" Web site and published as an ASAP article. Note that technical editing may introduce minor changes to the manuscript text and/or graphics which could affect content, and all legal disclaimers and ethical guidelines that apply to the journal pertain. ACS cannot be held responsible for errors or consequences arising from the use of information contained in these "Just Accepted" manuscripts. 


\section{INTRODUCTION}

Porous organic frameworks (POFs) consist of organic monomers linked by covalent bonds and have recently found many applications in the fields of catalysis and separation. ${ }^{1}$ Variation of the monomer units results in a wide range of materials with different pore size distributions and various functionalities. Owing to their high structural and functional tunability, POFs offer numerous advantages when compared to traditional inorganic materials. Thus, the development of new POF structures and their applications have been intensively researched over the last decade. Covalent triazine frameworks (CTFs) are among the most popular POF classes because they are composed of rigid conjugated aromatic networks with high thermal and chemical stability as well as very high porosity. ${ }^{2}$ CTFs are synthesized from relatively cheap aromatic nitriles and are formed by Lewis acid-mediated nitrile trimerization $^{2-3}$ (Scheme 1), although alternative synthetic routes are also possible $^{3 \mathrm{~d}, 4}$.

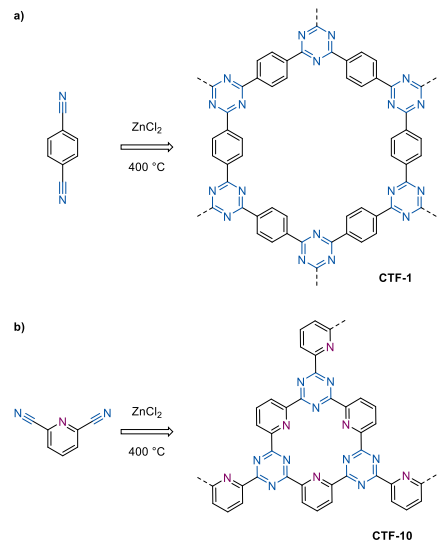

Scheme 1. Synthesis of CTFs from a) terephthalonitrile (1) and b) 2,6-pyridinedicarbonitrile (2) (idealized CTF structures).

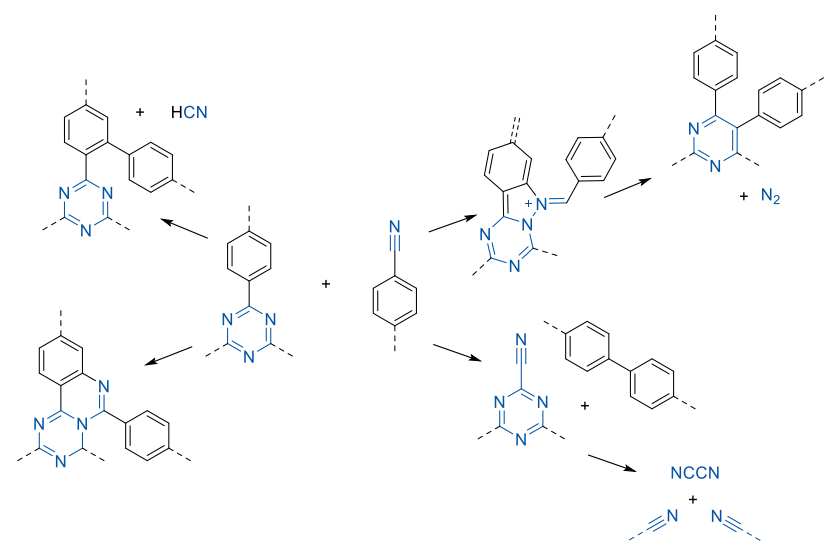

Scheme 2. Possible side reactions during the CTF synthesis at elevated temperatures.

Intrinsic nitrogen functionalities within the structure make CTFs very attractive materials for the coordination of metals ${ }^{5}$, as well as for selective adsorption ${ }^{3 a, 6}$. Since the first synthesis of CTFs using $\mathrm{ZnCl}_{2}$ as catalyst ${ }^{2}$, several attempts have been made to rationalize and optimize their synthesis. Extensive studies of the ionothermal synthetic conditions have shown that the framework porosity strongly depends on the synthesis temperature and the amount of catalyst used to promote the nitrile trimerization ${ }^{2,7}$. An increase of the polymer mesoporosity is observed with raising the synthesis temperature from 400 ${ }^{\circ} \mathrm{C}$ to $700{ }^{\circ} \mathrm{C}$, although at the expense of structure regularity. This effect can be a result of two factors: i) irreversible C-C and $\mathrm{C}-\mathrm{N}$ bond formation under Lewis-acid catalyzed conditions (e.g. Diels-Alder type reactions ${ }^{8}$, Scheme 2) and/or ii) carbonization of the material at high temperature. However, 
detailed studies have not been performed either on the nature of intermediate species or on the polymerization mechanism. Synthesis at lower temperature and extended time $\left(300^{\circ} \mathrm{C}\right.$ for 7 days) prevents these side reactions, but leads to a mixture of aryl-triazine oligomers (ATOs) ${ }^{9}$ with negligible to moderate porosity and surface area, rather than to a porous framework.

Most of the research dedicated to tuning the porosity by modifying the CTFs synthetic procedure has only been published on CTF-1, prepared from the monomer terephthalonitrile, 1 (Scheme 1a) ${ }^{7 b, 10}$. Access to CTFs with different functionalities is crucial for their application. CTF-10, synthesized from 2,6-pyridinedicarbonitrile (2), contains suitable pincerlike pyridinic-triazinic sites for metal coordination. The synthesis of CTF-10 compared to that of CTF-1 represents a more complicated process. Preferential zinc binding to the pyridine moieties promotes the activation of neighboring nitrile groups, causing back-donation from $\mathrm{Zn} d_{x z}$ and $d_{y z}$ orbitals to the anti-bonding $\pi^{*}$-orbital of the $\mathrm{C} \equiv \mathrm{N}$ group. This back donation can favor the trimerization reaction, as well as promote various side reactions. As a result of these additional side reactions, CTF-10 has significantly lower porosity compared to $\mathbf{C T F - 1}{ }^{7 \mathrm{c}}$.

It should be noted that most of CTFs are amorphous dark materials with strong absorbance in the spectral range of many common spectroscopic techniques. These features make the characterization of these materials difficult and hampers a better understanding of their structural and chemical properties. In contrast, the ATOs usually display a yellow color and can be characterized by methods involving visible and ultraviolet light irradiation ${ }^{9}$. Although ATOs can be considered as model compounds for CTF characterization, synthesis of CTFs from ATOs is still accompanied by some degree of amorphization giving rise to a variety of new carbon and nitrogen species that cannot be easily characterized. Alternative spectroscopies that use higher energy (XAS, XPS $)^{5 \mathrm{~b}, 11}$ or are based on magnetic properties (CP-MAS solid-state NMR $)^{6,10-11}$ become a common and useful tool for the challenging characterization of these materials. Moreover, due to the gamut of species present in the amorphous CTFs, interpretation of experimental data becomes challenging and demands in-depth study of the materials.

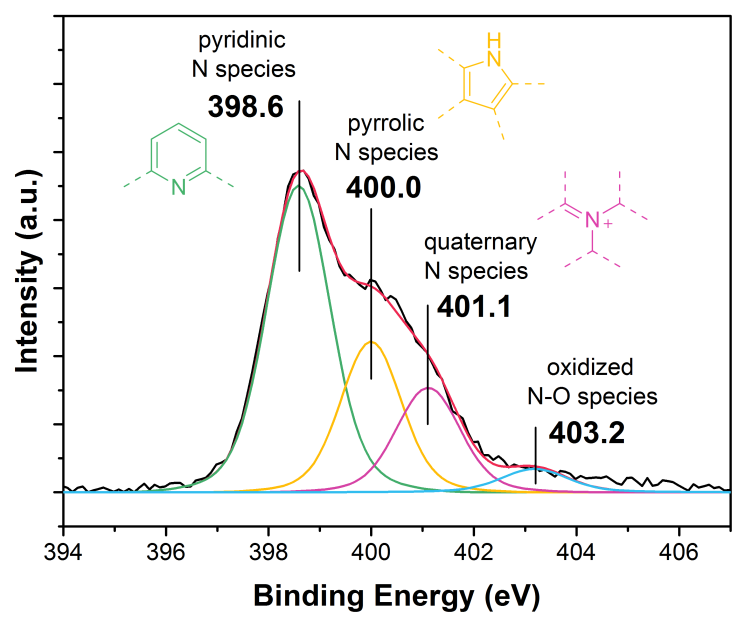

Figure 1. Deconvolution of the N1s spectrum of CTF-10 (ionothermal synthesis with 5 equivalents of $\mathrm{ZnCl}_{2}$ at $500{ }^{\circ} \mathrm{C}$ for $48 \mathrm{~h}$ ) with a typical peak assignment according to the literature.
The relatively broad range of N1s binding energies makes $\mathrm{X}$-ray photoelectron spectroscopy (XPS) a useful tool for characterization of species containing multiple nitrogen moieties in different electronic environments. XPS has already been successfully applied to a variety of carbon-nitrogen materials including carbon nitrides ${ }^{12}$, $\mathrm{N}$-functionalized amorphous carbons $^{13}$, N-doped carbon nanotubes and graphenes ${ }^{14}$ and $\mathrm{N}$ containing organic polymers ${ }^{15}$. However, non-systematic use of the method can provide only limited knowledge. As an example, the typical deconvolution ${ }^{11}$ of the N1s spectrum for CTF-10 (Fig.1) merely relies on assignments reported in the literature for amorphous carbons.

In this work, we show the opportunities that XPS offers for characterization of CTFs. By using XPS together with characterization of the textural properties we critically review CTF ionothermal synthesis and highlight the impact that different monomers, synthesis temperature and catalysts have on the final properties of these solids. Through a correlated study of model compounds, we are able to assign a number of nitrogen species that until now have been elusive and assigned only on basis of nitrogen containing organic polymers and carbonaceous supports.

\section{EXPERIMENTAL SECTION}

Synthesis of CTFs. The synthesis procedure for CTF materials was similar to the one reported by Kuhn et al ${ }^{2}$. Various dicarbonitrile monomers, trimerization catalysts (inorganic Lewis acids) and reaction temperatures were used for different samples. All samples reported in this work and details of their preparation are summarized in Table 1. In a typical synthesis, a quartz ampoule was charged with the mixture of corresponding nitriles and an anhydrous metal salt in a molar ratio of nitriles:chloride $=1: 5$ inside the glovebox. The ampoule was flame sealed and heated to the desired temperature with a heating rate of $1^{\circ} \mathrm{C} \mathrm{min}^{-1}$, kept at this temperature for 48 hours and then cooled to room temperature. The product was consequently washed in $15 \% \mathrm{HCl}$ solution at $100{ }^{\circ} \mathrm{C}$, in $15 \%$ $\mathrm{NH}_{4} \mathrm{OH}$ solution at $60{ }^{\circ} \mathrm{C}$, in $\mathrm{H}_{2} \mathrm{O}$ at $100{ }^{\circ} \mathrm{C}$ and finally in THF at $60{ }^{\circ} \mathrm{C}$ overnight. The resulting powder was dried under vacuum at $180{ }^{\circ} \mathrm{C}$ overnight. The overall yield was typically in the range of $90-98 \%$.

Synthesis of model compounds. Complexes 4-Cu and 5Cu (Fig. 2) were prepared from commercially available 2,4,6triphenyl-1,3,5-triazine (4) and 2,4,6-tris(2-pyrydyl)-1,3,5triazine (5), respectively. In a typical synthesis, a mixture $1: 1$ molar ratio of the corresponding ligand and the tetrakis(acetonitrile)copper(I) hexafluorophosphate salt $\left(\mathrm{Cu}(\mathrm{MeCN})_{4} \mathrm{PF}_{6}\right)$ was dissolved in methanol and set under reflux at $80^{\circ} \mathrm{C}$ overnight. The resulting solids were filtered and dried in air at $80^{\circ} \mathrm{C}$.

Synthesis of Cu-CTF-10. $496 \mathrm{mg}$ of CTF-10 and $250 \mathrm{mg}$ of copper(II) acetate monohydrate were mixed in $20 \mathrm{~mL}$ of methanol under constant stirring with reflux at $80^{\circ} \mathrm{C}$ overnight. The resulting black solid was filtered and dried in air at $80{ }^{\circ} \mathrm{C}$.

X-ray photoelectron spectroscopy. XPS measurements were performed on a $K$-alpha Thermo Fisher Scientific spectrometer using a monochromatic $\mathrm{Al} K \alpha \mathrm{X}$-ray source. In a typical experiment, the sample powder was mounted on a double-faced conducting carbon tape (NEM TAPE, Nisshin EM Co., Ltd.), attached to a sample holder. The holder with 
the mounted samples was loaded into the sample loading chamber and set under vacuum to reach $2 \cdot 10^{-7}$ mbar. At this pressure, the sample holder was transferred into the analysis chamber and set to reach c.a. $10^{-8}$ mbar. The X-ray gun was operated at $3 \mathrm{~mA}$ and $12 \mathrm{kV}$ and the spot size was set to 400 $\mu \mathrm{m}$. Precision spectra of the core photoelectron lines were registered with $0.1 \mathrm{eV}$ energy step using constant analyzer pass energy of $50 \mathrm{eV}$. The measurements were performed at ambient temperature, the chamber pressure during the measurement was about $10^{-7}$ mbar. A flood gun was used for charge compensation.

Table 1. Synthesis conditions and textural properties of CTFs

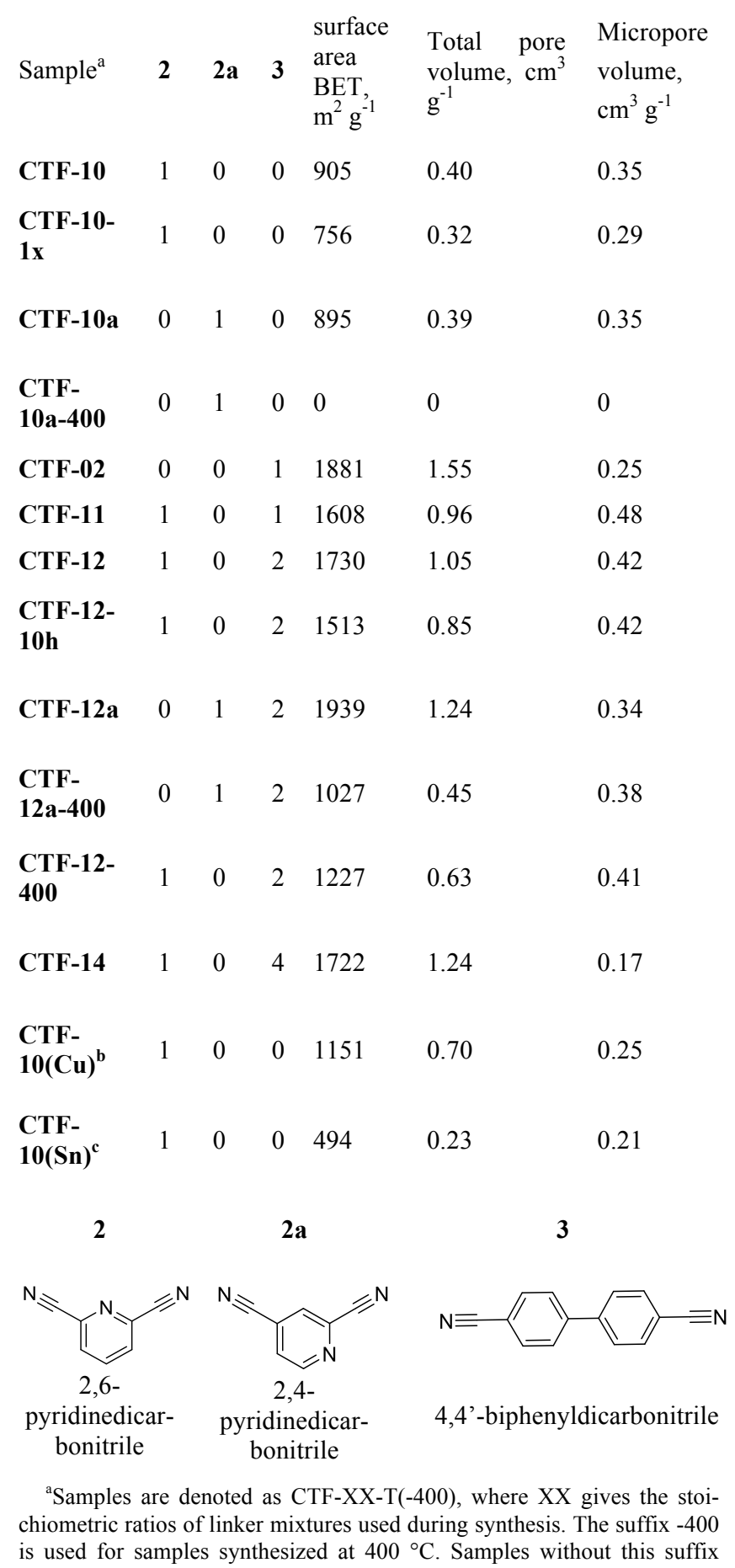

were synthesized at $500^{\circ} \mathrm{C}$.

${ }^{\mathrm{b}} \mathrm{Synthesized} \mathrm{using} \mathrm{CuCl}$ as ionothermal Lewis acid catalyst.

${ }^{\text {c}}$ Synthesized using $\mathrm{SnCl}_{2}$ as ionothermal Lewis acid catalyst.

All the measured spectra were corrected by setting the reference binding energy of carbon $(\mathrm{C} 1 \mathrm{~s})$ at $285.0 \pm 0.025 \mathrm{eV}$. Spectra were analyzed using the Thermo Avantage software package. Background subtraction was done using the setting "SMART" (based on the Shirley background with the additional constraint that the background should not be of a greater intensity than the actual data at any point in the region). The deconvolution of spectra was performed using a mixed GaussLorentz function where the Lorentzian contribution was set to $20 \%$ for $\mathrm{C} 1 \mathrm{~s}, \mathrm{~N} 1 \mathrm{~s}$ and $\mathrm{O} 1 \mathrm{~s}$ and to $30 \%$ for $\mathrm{Cu} 2 \mathrm{p}, \mathrm{Zn} 2 \mathrm{p}, \mathrm{Sn} 2 \mathrm{p}$. Quantification was done using the Scoffield sensitivity factors. Difference in depth of analysis for different photoelectron lines was accounted using the TPP-2M method ${ }^{16}$.

Nitrogen adsorption measurements were performed using a Tristar II 3020 Micromeritics instrument at $77 \mathrm{~K}$. Samples were activated under $\mathrm{N}_{2}$ flow for at least $13 \mathrm{~h}$ at $150{ }^{\circ} \mathrm{C}$. Pore size distribution, pore volume and surface area were calculated using Microactive Version 3.00 software.

\section{RESULTS AND DISCUSSION}

The N1s spectra of CTFs normally consist of a peak with $\mathrm{BE} \approx 398.5 \mathrm{eV}$, usually attributed to "pyridine-like" aromatic nitrogen, and a broader shoulder at higher binding energy, considered to arise from partial framework decomposition. Common deconvolution of the N1s line is given by a "pyridine-like" peak and three other peaks to fit the shoulder (Fig. $1)$. The designation of these peaks - "pyrrol-like" (BE $\approx 400$ $\mathrm{eV})$, "quaternary" (BE $\approx 401 \mathrm{eV}$ ) and "oxygenated $(\mathrm{N}-\mathrm{O})$ " $(\mathrm{BE}>402 \mathrm{eV})$ nitrogen species - is based on the nomenclature used in the literature for $\mathrm{N}$-doped carbons ${ }^{13 b}$. Coordination of metals to CTFs leads to increasing of the peak between $399.5-400 \mathrm{eV}$. This effect is often attributed to the interaction of the nitrogen binding sites of CTFs with metals. However, it was never unambiguously assigned to a metal-framework interaction $^{5 \mathrm{~b}-\mathrm{d}, 11}$.

To assign the different signals arising from $\mathrm{N}$ species in the CTFs, we investigated some reference compounds. 2,4,6triphenyl-1,3,5-triazine (4) and 2,4,6-tris(2-pyrydyl)-1,3,5triazine (5) were chosen as model compounds as they represent the structural units of CTF-1 and CTF-10 (Scheme 1). 1,3,5-triazine (6) without any substituents was also measured for comparison. To have a reference for non-reacted nitrile groups, we have also investigated 3 and 1,2,4,5tetracyanobenzene (7) with XPS. Measurements of other nitrile-containing monomers reported in this work were not possible due to their low adhesion towards the supporting material (carbon tape) and higher volatility in high vacuum conditions.

The results show that the exact position of the N1s line from triazinic species strongly depends on the nature of the substituents of the ring (Figure 2). While the non-substituted triazine (6) is characterized by a peak with $\mathrm{BE}=400.3 \mathrm{eV}$, substitution of hydrogen atoms for electron-donating phenyl rings in the triazine ring (4) shifts the peak to significantly lower binding energy, $397.9 \mathrm{eV}$. When pyridinic groups are present (compound 5), two different nitrogen species in XPS can be expected. However, in the N1s line of $\mathbf{5}$ only one peak, shifted to 
higher binding energy $(398.7 \mathrm{eV})$, is observed. Full width at half-maximum (FWHM) of this peak is the same as for $\mathbf{4}$ $(\mathrm{FWHM}=1.3 \mathrm{eV})$. Thus, 5 represents a fully conjugated aromatic system where the nitrogen moieties in the triazine unit and pyridine rings result in electronically similar species, and likely the peak at $398.7 \mathrm{eV}$ is a superposition of pyridinic and triazinic species with very close binding energy. Taking into account that pyridine and triazine are 6-membered nitrogen-containing aromatic heterocycles with each nitrogen atom contributing only one electron into the $\pi$-aromatic ring, we further denote this peak as N6 peak.

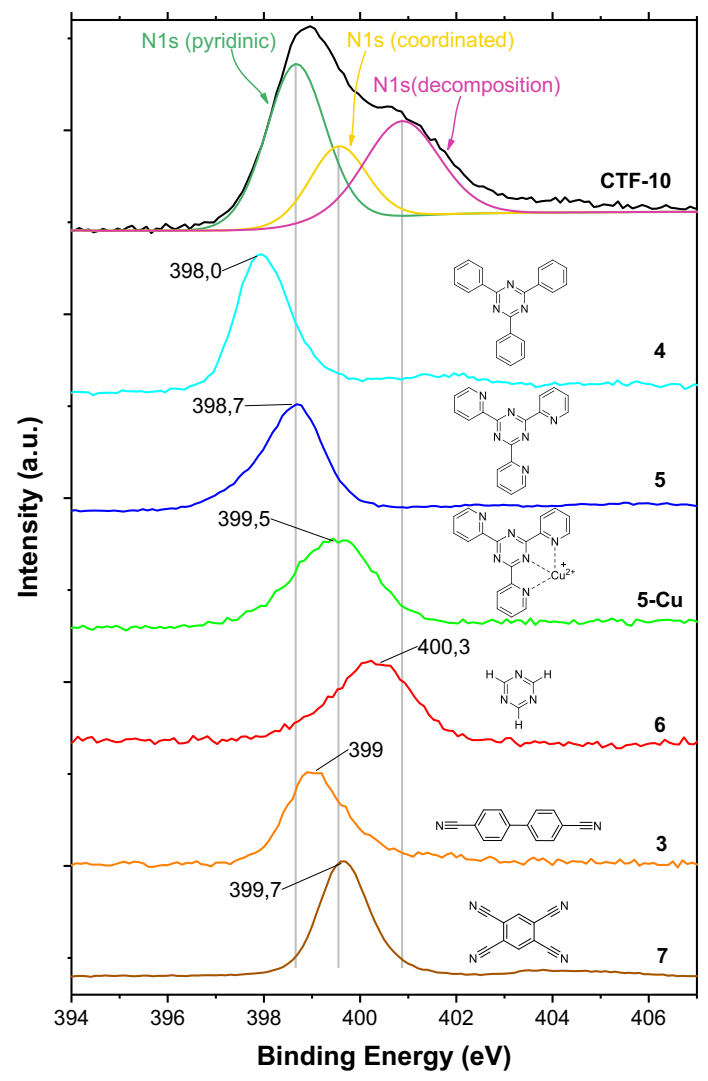

Figure 2. N1s XPS spectra of CTF-10 compared to that of the model compounds: phenyl substituted triazine (4), pyridine functionalized triazine (5), the $\mathrm{Cu}$ complex $\mathbf{5}-\mathbf{C u}$, non-functionalized triazine (6), and 4,4'-biphenyldicarbonitrile (3) and 1,2,4,5tetracyanobenzene (7) monomers for CTF synthesis. The gray lines indicate the maxima of the three peaks obtained for the deconvolution of CTF-10 N1s line (BE= 398.7, 399.6, and 400.9 $\mathrm{eV})$.

To investigate how metal coordination affects the N1s XPS spectra of CTFs, we also prepared the Cu complexes of $\mathbf{4}$ and $\mathbf{5}$ (denoted as 4-Cu and 5-Cu, respectively). Mixing of $\mathbf{4}$ with $\mathrm{Cu}(\mathrm{MeCN})_{4} \mathrm{PF}_{6}$ in solution does not result in any color change and the position of the N1s peak does not shift. In contrast, mixing of a $\mathrm{Cu}$ salt with $\mathbf{5}$ leads to rapid color change of the solution to dark red, and the N6 peak in the N1s spectrum of the resulting complex clearly shifts to higher binding energy $(399.5 \mathrm{eV})$. Thus, the 1,3,5-triazinic groups within the CTF structure have poor coordinating properties for transitional metal ions, but polydentate pyridine-triazine species are advantageous for metal coordination.
Discrimination between the N6 and unreacted nitrile species with XPS turns out to be more complicated. N1s XPS spectrum of 7 shows a single peak at $399.65 \mathrm{eV}$, that overlaps with coordinated N6 peak. However, C1s XPS spectrum of 7 shows an intense peak at $286.8 \mathrm{eV}$, that can be used for distinguishing these species (Figure S1). In case of 3 the nitrile peak appears in N1s XPS spectrum at $399.0 \mathrm{eV}$, that is located in between coordinated and non-coordinated N6 species, making it difficult to identify. Thus, in most of cases the free nitrile peak in N1s line will be hidden by N6 peaks, so the fraction of unreacted nitriles could be estimated only from the peak at approx. $287 \mathrm{eV}$ in $\mathrm{C} 1 \mathrm{~s}$ line.

Using the peak positions from model compounds, we deconvoluted the N1s line of samples $\mathbf{C T F}-10$ and $\mathrm{Cu}$ coordinated to this framework (Cu-CTF-10, Fig. 3). Fitting resulted in four peaks $(398.7,399.5 \pm 0.1,401.0 \pm 0.1$ and $403.0 \pm 0.2 \mathrm{eV})$ attributed to free N6 species, coordinated N6 species, products of partial CTF decomposition and oxidized $\mathrm{N}-\mathrm{O}$ species, respectively. Including several peaks for decomposition products was making the fitting too ambiguous, so we decided to use one broad peak at $\approx 401 \mathrm{eV}$ for all species arising from CTF degradation. The increased intensity of the peak at 399.5 $\mathrm{eV}$ demonstrates that metal coordination contributes to this peak and proves the applicability of the proposed deconvolution model. Using this model, we investigate the influence of monomer composition, synthesis temperature and trimerization catalyst in CTFs materials.

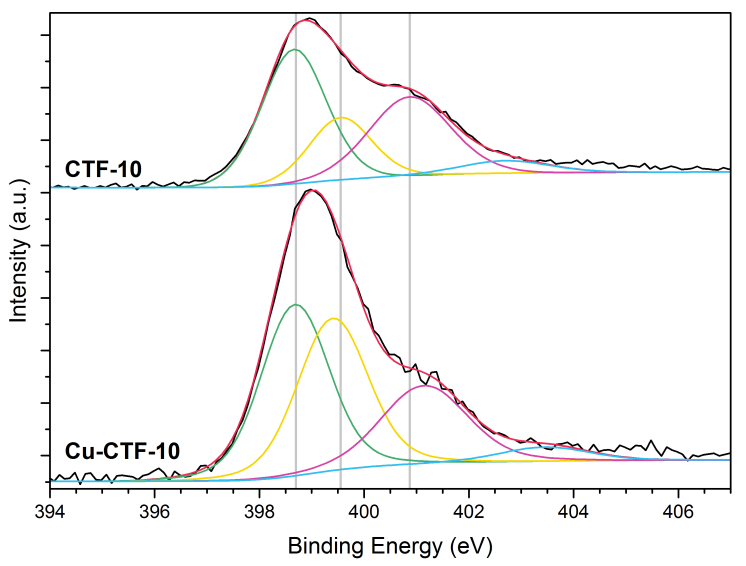

Figure 3. Deconvoluted N1s XPS spectra of CTF-10 and CuCTF-10. The gray lines indicate the maxima of the three peaks obtained for the deconvolution of CTF-10 N1s line $(\mathrm{BE}=398.7$, 399.6, and $400.9 \mathrm{eV})$.

Coordination properties of the monomer during synthesis. CTF-10 is a microporous material and it is possible to introduce mesoporosity either by: (i) increasing the synthesis temperature or (ii) introduction of different monomers with distinct topologies (e.g. 4,4'-biphenyldicarbonitrile, 3 ) to the reaction mixture ${ }^{5 \mathrm{c}, 5 \mathrm{~d}, 6 \mathrm{a}, 7 \mathrm{a}}$. To analyze the impact of the monomer ratio during synthesis and how this reflects on the CTF XPS N1s line, we prepared a series of CTFs with different monomer ratios (2 to 3 ) in the reaction mixture $-1: 1,1: 2$ and 1:4 ratios, denoted as CTF-11, CTF-12 and CTF-14, respectively (see Table 1). The CTF obtained only from monomer 3 was also prepared for comparison (denoted as CTF-02). 
XPS C1s spectra of CTF samples (Figure S2) were fitted using four peaks with binding energies of $285.0 \mathrm{eV}$ (adventitious carbon/C-C bonds), $286.4 \pm 0.2 \mathrm{eV}$ (C-O groups), $287.5 \pm 0.2 \mathrm{eV}$ $(\mathrm{C} \equiv \mathrm{N}$ groups) and $289.0 \pm 0.1 \mathrm{eV}$ (O-C=O groups). The ratio of $\mathrm{C} \equiv \mathrm{N}$ to $\mathrm{C}-\mathrm{O}$ peak areas was nearly constant, and the $\mathrm{C} \equiv \mathrm{N}$ peak area was around $5 \%$ of $\mathrm{C} 1 \mathrm{~s}$ line for all CTFs (Table S2).
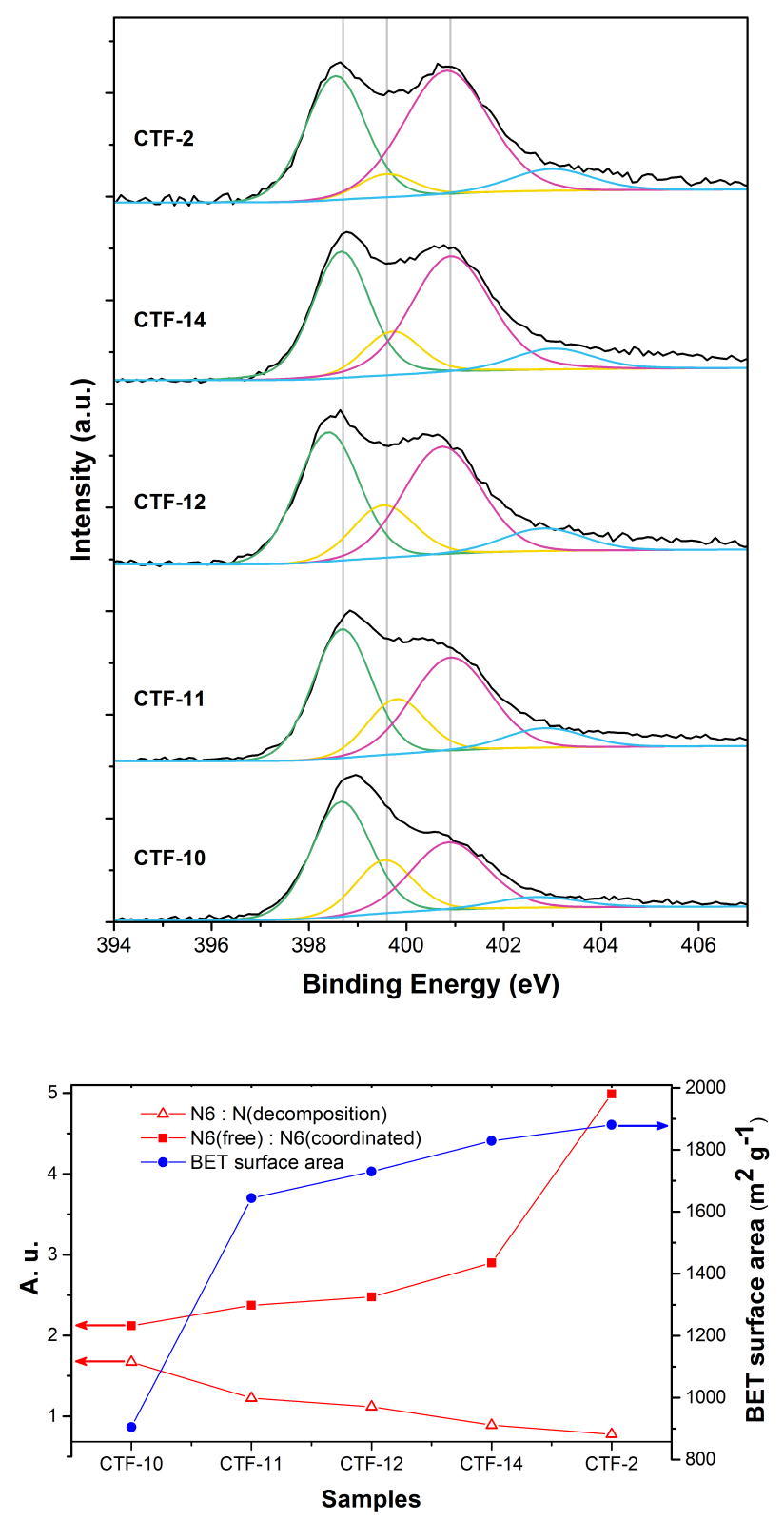

Figure 4. a) N1s XPS spectra and b) evolution of BET surface area, ratio of $\mathrm{N} 6$ to decomposition nitrogen species and ratio of free N6 to coordinated N6 species for CTFs prepared from mixtures of monomers $\mathbf{2}$ and $\mathbf{3}$ with different ratios. Nomenclature according to Table 1 .

According to Zn2p XPS data, all CTF samples contain residual amounts of zinc (see Table S3). This observation is supported by the results of elemental analysis for selected samples (2.87 wt \% of $\mathrm{Zn}$ in CTF-10, $0.3 \mathrm{wt} \%$ of $\mathrm{Zn}$ in CTF12). Content of zinc roughly correlates with the content of nitrogen for all samples, thus, coordinated N6 species are expected to be present in all CTFs.

The XPS N1s spectra for these CTF series are shown in Fig.4a. When using a greater number of equivalents of $\mathbf{3}$, a relative decrease of the total amount of N6 species in CTFs and an increased ratio of free to coordinated N6 species is observed (Figure 4b). Although there is a difference in the N6 peak position in $\mathbf{4}$ and $\mathbf{5}$ and the same shift can be expected when increasing equivalents of $\mathbf{3}$, the N6 peak shows a negligible shift among all samples, including CTF-02. This suggests that the electron donation of each biphenyl unit in the CTFs structure is lower compared to that in $\mathbf{4}$, where the phenyl rings are connected to only one triazine unit.

According to $\mathrm{N}_{2}$ adsorption measurements (Fig. 4b, Fig S3), higher loading of $\mathbf{3}$ significantly increases the surface area and mesoporosity of CTFs. Fig. 4 shows that these changes are accompanied by an increase of the CTF decomposition products peak in the N1s XPS spectra. Thus, biphenyl groups of $\mathbf{3}$ favor the reactions of CTF decomposition (Scheme 2), and the increase of porosity is achieved at the expenses of the total number of sites available for coordination within the structure. However, it is not clear whether the framework decomposition or the bigger size of $\mathbf{3}$ has a greater effect on the CTF porous structure.

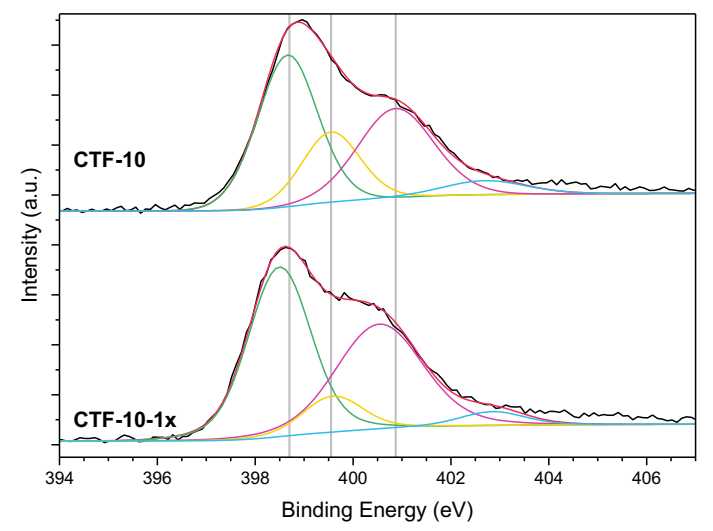

Figure 5. N1s XPS spectra of CTF-10 and CTF-10-1x.

Influence of the catalyst amount. It was shown for CTF-1, that decomposition of CTFs depends strongly on the amount of catalyst used for the synthesis. For that framework the most crystalline sample was prepared with the ratio of monomer: $\mathrm{ZnCl}_{2}=1: 1^{7 \mathrm{c}}$. However, in case of CTF-10 the optimal amount of catalyst can be different due to the presence of coordinating pyridinic groups within the framework. In order to investigate this, we have prepared $\mathbf{C T F}-\mathbf{1 0}$ with 1:1 catalyst loading (further denoted as CTF-10-1x). Comparison of C1s XPS spectra (Fig. S4) shows that CTF-10-1x has higher content of free $\mathrm{C} \equiv \mathrm{N}$ groups than $\mathbf{C T F}-\mathbf{1 0}(8.1 \%$ and $5.7 \%$ of total $\mathrm{C} 1 \mathrm{~s}$ line area, respectively). The ratio of $\mathrm{N} 6$ species to decomposition products, obtained from deconvolution of N1s XPS spectra (Fig. 5), was found equal to 1.94 and 1.41 for CTF-10 and CTF-10-1x, respectively. This indicates that reactions of CTF decomposition take place both in case of $1: 1$ and 5:1 ratios of $\mathrm{ZnCl}_{2}$ :monomer, but for 1:1 loading the CTF synthesis is not complete and still a lot of free nitrile groups are present after synthesis. Thus, the optimal amount of $\mathrm{ZnCl}_{2}$ catalyst for CTF-10 synthesis is different from equimolar, 
probably due to the presence of coordinating pyridinic sites in the monomer structure.

Influence of the reaction time. In order to investigate how CTF properties depend on the reaction time we have run the synthesis of CTF-12 for $10 \mathrm{~h}$ and for $48 \mathrm{~h}$ (these samples are further denoted as CTF-12-10h and CTF-12, respectively). N2 adsorption measurements (Fig. S7) show that CTF-12 has higher BET surface area, but the micropore volume is the same for both samples, indicating that porosity of CTF-12 increases only due to the framework decomposition. This is supported by C1s XPS spectra (Fig. S6), where content of unreacted $\mathrm{C} \equiv \mathrm{N}$ groups is close for both samples, meaning that nitrile trimerization reaction is complete after $10 \mathrm{~h}$. N1s XPS spectra (Fig. 6) show the same ratio of N6 to decomposition species for both samples, but the total content of nitrogen decreases with increasing reaction time. This indicates that decomposition of CTF at $500^{\circ} \mathrm{C}$ mainly occurs via formation of $\mathrm{C}-\mathrm{C}$ bonds with elimination of nitrogen in a form of volatile $\mathrm{N}$-containing compounds ( $\mathrm{HCN}, \mathrm{NCCN}$, etc).

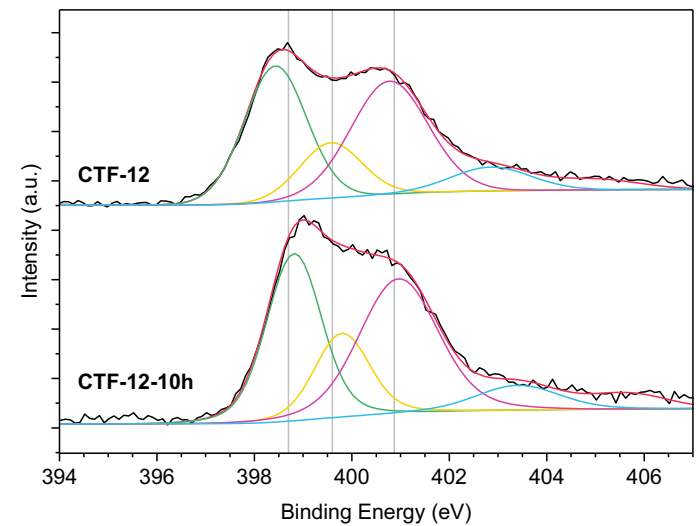

Figure 6. N1s XPS spectra of CTF-12 and CTF-12-10h.

Influence of synthesis temperature. The increase of synthesis temperature from $400^{\circ} \mathrm{C}$ in $\mathbf{C T F}-\mathbf{1 2 - 4 0 0}$ to $500^{\circ} \mathrm{C}$ in CTF-12 leads to an increase of decomposition products that accounts for more than half of the total amount of nitrogen species (Fig. 7). Total nitrogen content (Table S3) and C1s line shape (Fig. S8) do not change at higher temperature. $\mathrm{N}_{2}$ adsorption measurements (Fig. S7) show that the micropore volume remains unchanged, but the mesopore volume becomes 3 times higher in CTF-12 compared to CTF-12-400. This proves that the mesoporosity of CTFs originates from thermal decomposition of the framework and correlates with the higher intensity of the decomposition product peak in the XPS N1s line.

Influence of monomer structure. To understand the role of the monomer structure during CTF synthesis we have prepared CTF-10a using an isomer of 2 - 2,4-pyridinedicarbonitrile (2a, see Table 1). This monomer has only one of the two nitrile groups near the pyridinic nitrogen. The second nitrile group is located far from the pyridinic nitrogen, so it is expected to be closer in reactivity to the nitrile groups of $\mathbf{1}$ and $\mathbf{3}$. Having two different nitrile groups, a two-step reaction mechanism, where the trimerization rate of nitriles in ortho-position to the pyridinic nitrogen can be faster to that in para-position, can be expected. As it was already discussed above, close proximity of nitrile groups to the pyridinic $\mathrm{N}$ atom in $\mathbf{2}$ should promote the synthesis of CTF and potentially the decomposition reactions. The N1s line of the sample synthesized at $400^{\circ} \mathrm{C}(\mathbf{C T F}-10 a-400)$ shows one main peak with $\mathrm{BE}=398.9$ $\mathrm{eV}$ and a negligible amount of decomposition products (Fig. 8). Binding energy of $398.9 \mathrm{eV}$ matches perfectly with the values observed for $\mathbf{3}$, that contains free nitrile groups. Increase of the free $\mathrm{C} \equiv \mathrm{N}$ peak is also observed in C1s XPS line (Fig. S9). Adsorption measurements show that CTF-10a-400 has no porosity. Thus, this suggests that when using 2a only oligomers are formed rather than an extended CTF framework. This also confirms that the nitrile groups in ortho and para position of the pyridine ring have significantly different reactivity in trimerization reaction.

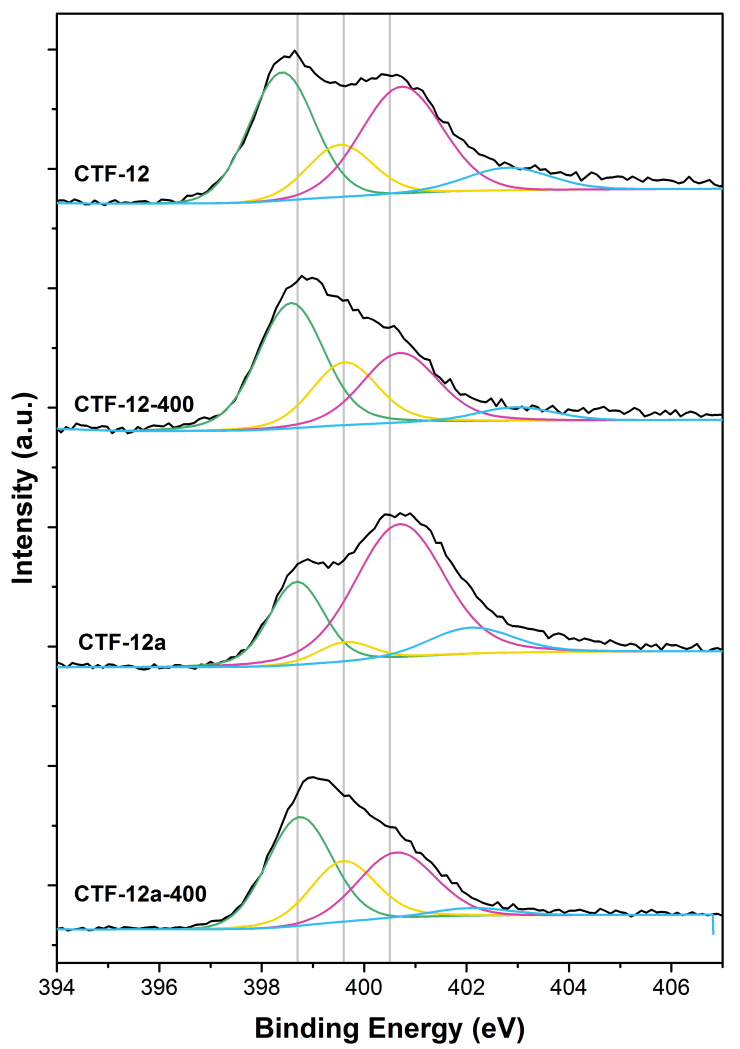

Figure 7. N1s XPS spectra of CTF-12, CTF-12-400, CTF-12a, and CTF-12a-400.

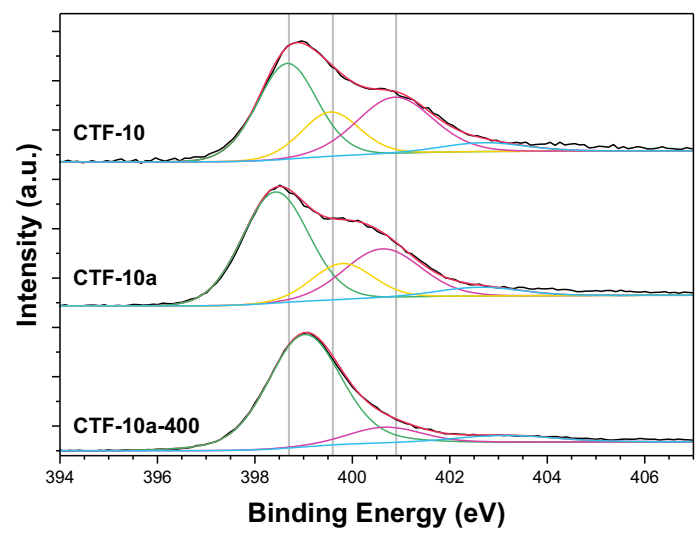


Figure 8. N1s XPS spectra of CTF-10, CTF-10a and CTF10a-400.

Use of 2a for synthesis of CTF-12a-400 leads to lower surface area and mesopore volume compared to CTF-12-400. N1s XPS spectra for these two samples are very similar (Fig. 7), but $\mathrm{C} 1 \mathrm{~s}$ XPS spectra show increase of $\mathrm{C} \equiv \mathrm{N}$ peak in case of CTF-12a-400 (Fig. S8). On the contrary, CTF-12a, prepared at $500^{\circ} \mathrm{C}$, has higher mesoporosity and surface area than $\mathbf{C T F}$ 12. XPS characterization of CTF-12a demonstrates that it has significantly lower concentration of N6 species and higher concentration of framework decomposition products in the N1s line compared to CTF-12.

Use of $\mathbf{2 a}$ instead of $\mathbf{2}$ for CTF synthesis hampers the framework formation at $400^{\circ} \mathrm{C}$ and promotes the decomposition reactions at $500^{\circ} \mathrm{C}$. This shows that uniform activation of nitrile groups within the monomer is critical for the formation of the framework and proves that the formation of $\mathrm{Zn}$ monomer complexes has an important structure-directing role.

Influence of the catalyst nature. Complexation of $\mathrm{Zn}$ to the monomer 2 promotes the activation of the nitrile groups and the CTF formation. The use of a Lewis acid that is able to form a more stable complex with 2 than that of $\mathrm{ZnCl}_{2}$, can be beneficial for the synthesis procedure. The use of $\mathrm{CuCl}$ as catalyst for $\mathrm{CTF}$ synthesis is attractive as it forms much stronger complexes with 2 than $\mathrm{ZnCl}_{2}{ }^{17}$. A drawback of using $\mathrm{CuCl}$ is its higher melting temperature and higher oxidation potential compared to $\mathrm{ZnCl}_{2}$. Another promising catalyst for CTF synthesis is $\mathrm{SnCl}_{2}$ because has lower melting temperature than $\mathrm{ZnCl}_{2}$ and has an intermediate oxidation potential compared to $\mathrm{CuCl}$ and $\mathrm{ZnCl}_{2}$ (Table 2).

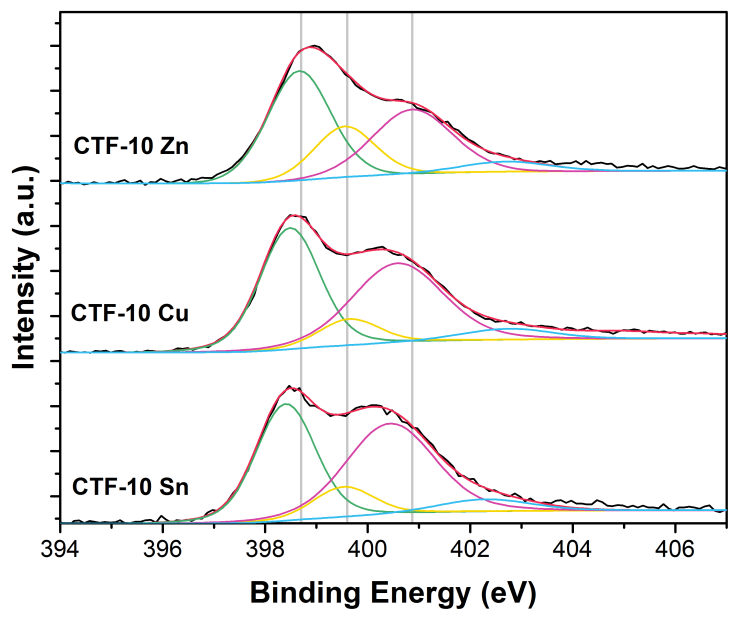

Figure 9. N1s XPS spectra of CTF samples prepared from 2 at $500^{\circ} \mathrm{C}$ using $\mathrm{ZnCl}_{2}, \mathrm{CuCl}$ and $\mathrm{SnCl}_{2}$ as trimerization catalysts.

Table 2. Properties of some inorganic Lewis acids

$\begin{array}{ccc}\text { Lewis acid } & \text { Melting T, }{ }^{\circ} \mathrm{C} & \begin{array}{c}\text { Standard oxidation potential } \\ \left(\mathrm{M}^{\mathrm{n}+}+\mathrm{ne}=\mathrm{M}^{0}\right)\end{array} \\ \mathrm{ZnCl}_{2} & 290 & -0,7628 \\ \mathrm{CuCl} & 426 & 0,521 \\ \mathrm{SnCl}_{2} & 247 & -0,14\end{array}$

Fig. S10 represents $\mathrm{N}_{2}$ adsorption isotherms for CTF-10 samples prepared at $500^{\circ} \mathrm{C}$ using $\mathrm{ZnCl}_{2}, \mathrm{CuCl}$ and $\mathrm{SnCl}_{2}$ as trimerization catalysts (denoted as CTF-10(Zn), CTF-10(Cu) and CTF-10(Sn), respectively). CTF-10(Cu) is significantly more porous than CTF-10(Zn) and even exhibits some mesoporosity. Comparison of XPS N1s spectra (Fig. 9) shows that CTF-10(Cu) and CTF-10(Sn) have a smaller fraction of N6 species and relatively higher content of framework decompo-

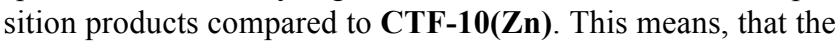
increased porosity of CTF-10(Cu) is a consequence of the partial decomposition of the framework. Interestingly, the sealed ampoule that was used for the CTF-10(Cu) synthesis was coated with a thin layer of metallic copper after $48 \mathrm{~h}$ of reaction, indicating the reduction of $\mathrm{CuCl}$ during synthesis. On the contrary, when using $\mathrm{SnCl}_{2}$ the surface area decreases. Quantification of XPS data shows that tin to nitrogen ratio in CTF-10(Sn) is significantly higher than zinc to nitrogen ratio in CTF-10(Zn) and copper to nitrogen ratio in CTF-10(Cu) $(0.20,0,05$ and 0,02 , respectively), suggesting that the pores of CTF-10(Sn) can be partially blocked by fully encapsulated residuals of the catalyst.

The use of Lewis acids other than $\mathrm{ZnCl}_{2}$ as catalysts for CTF synthesis can be beneficial for the one-step introduction of the desired metal (e.g. copper) into the structure of CTF. However, under ionothermal conditions many metal salts can easily oxidize organics, thus promoting decomposition of the framework and an increase in mesoporosity.

\section{CONCLUSIONS}

Synthesis of CTFs proceeds through a delicate interplay between framework formation via nitrile trimerization and framework decomposition through C-C coupling. By combining the results of a thorough XPS study with the textural properties of CTFs synthesized under different conditions, we show that decomposition of CTFs increases the surface area and mesoporosity of the material, but decreases the relative amount of accessible nitrogen species. Increase of synthesis time and temperature and use of oxidizing compounds as trimerization catalysts promote the decomposition reactions. Use of monomers that form more stable complexes with Lewis acids favors the $\mathrm{CTF}$ formation and reduces the rate of framework decomposition.

\section{ASSOCIATED CONTENT}

\section{Supporting Information}

The Supporting Information is available free of charge on the ACS Publications website.

\section{AUTHOR INFORMATION}

\section{Corresponding Author}

*jorge.gascon@kaust.edu.sa

\section{ACKNOWLEDGMENT}

The authors gratefully acknowledge funding from the Dutch National Organization for Scientific Research (NWO), VIDI grant agreement 723.012.107 - MetMOFCat.

\section{REFERENCES}


1. (a) Qiu, S.; Ben, T., Porous Polymers: Design, Synthesis and Applications. The Royal Society of Chemistry: Thomas Graham House, Science Park, Milton Road, Cambridge CB4 0WF, UK, 2015; (b) Zhu, G.; Ren, H., POROUS ORGANIC FRAMEWORKS Design, Synthesis and Their Advanced Applications. Springer Berlin Heidelberg: 2015.

2. Kuhn, P.; Antonietti, M.; Thomas, A., Porous, covalent triazine-based frameworks prepared by ionothermal synthesis. Angewandte Chemie - International Edition 2008, 47 (18), 3450-3453.

3. (a) Zhu, X.; Tian, C.; Mahurin, S. M.; Chai, S.-H.; Wang, C.; Brown, S.; Veith, G. M.; Luo, H.; Liu, H.; Dai, S., A Superacid-Catalyzed Synthesis of Porous Membranes Based on Triazine Frameworks for $\mathrm{CO} 2$ Separation. Journal of the American Chemical Society 2012, 134 (25), 10478-10484; (b) Ren, S.; Bojdys, M. J.; Dawson, R.; Laybourn, A.; Khimyak, Y. Z.; Adams, D. J.; Cooper, A. I., Porous, fluorescent, covalent triazine-based frameworks via room-temperature and microwave-assisted synthesis. Advanced Materials 2012, 24 (17), 2357-2361; (c) Miller, G. H. NITRILE TRIMERIZATION PROCESS. 1972; (d) Meier, C. B.; Sprick, R. S.; Monti, A.; Guiglion, P.; Lee, J.S. M.; Zwijnenburg, M. A.; Cooper, A. I., Structureproperty relationships for covalent triazine-based frameworks: The effect of spacer length on photocatalytic hydrogen evolution from water. Polymer.

4. $\quad$ Byun, J.; Patel, H. A.; Thirion, D.; Yavuz, C. T., Reversible water capture by a charged metal-free porous polymer. Polymer (United Kingdom) 2017, 126, 308-313.

5. (a) Palkovits, R.; Antonietti, M.; Kuhn, P.; Thomas, A.; Schüth, F., Solid catalysts for the selective low-temperature oxidation of methane to methanol. Angewandte Chemie - International Edition 2009, 48 (37), 6909-6912; (b) Kamiya, K.; Kamai, R.; Hashimoto, K.; Nakanishi, S., Platinum-modified covalent triazine frameworks hybridized with carbon nanoparticles as methanol-tolerant oxygen reduction electrocatalysts. Nature Communications 2014, 5; (c) Bavykina, A. V.; Goesten, M. G.; Kapteijn, F.; Makkee, M.; Gascon, J., Efficient production of hydrogen from formic acid using a Covalent Triazine Framework supported molecular catalyst. ChemSusChem 2015, 8 (5), 809-812; (d) Rozhko, E.; Bavykina, A.; Osadchii, D.; Makkee, M.; Gascon, J., Covalent organic frameworks as supports for a molecular $\mathrm{Ni}$ based ethylene oligomerization catalyst for the synthesis of long chain olefins. Journal of Catalysis 2017, 345, 270280; (e) Yoshioka, T.; Iwase, K.; Nakanishi, S.; Hashimoto, K.; Kamiya, K., Electrocatalytic Reduction of Nitrate to Nitrous Oxide by a Copper-Modified Covalent Triazine Framework. The Journal of Physical Chemistry C 2016, 120 (29), 15729-15734.

6. (a) Dey, S.; Bhunia, A.; Breitzke, H.; Groszewicz, P. B.; Buntkowsky, G.; Janiak, C., Two linkers are better than one: enhancing $\mathrm{CO} 2$ capture and separation with porous covalent triazine-based frameworks from mixed nitrile linkers. Journal of Materials Chemistry A 2017, 5 (7), 3609-3620; (b) Hug, S.; Stegbauer, L.; Oh, H.; Hirscher, M.; Lotsch, B. V., Nitrogen-Rich Covalent
Triazine Frameworks as High-Performance Platforms for Selective Carbon Capture and Storage. Chemistry of Materials 2015, 27 (23), 8001-8010.

7. (a) Kuhn, P.; Forget, A.; Hartmann, J.; Thomas, A.; Antonietti, M., Template-free tuning of nanopores in carbonaceous polymers through lonothermal synthesis. Advanced Materials 2009, 21 (8), 897-901; (b) Kuhn, P.; Forget, A.; Su, D.; Thomas, A.; Antonietti, M., From microporous regular frameworks to mesoporous materials with ultrahigh surface area: Dynamic reorganization of porous polymer networks. Journal of the American Chemical Society 2008, 130 (40), 13333-13337; (c) Kuhn, P.; Thomas, A.; Antonietti, M., Toward tailorable porous organic polymer networks: A high-temperature dynamic polymerization scheme based on aromatic nitriles. Macromolecules 2009, 42 (1), 319-326.

8. Konovalov, A. I.; Kiselev, V. D., Diels - Alder reaction: Effect of internal and external factors on the reactivity of diene - dienophile systems. Russian Chemical Bulletin 2003, 52 (2), 293-311.

9. Schwinghammer, K.; Hug, S.; Mesch, M. B.; Senker, J.; Lotsch, B. V., Phenyl-triazine oligomers for light-driven hydrogen evolution. Energy and Environmental Science 2015, 8 (11), 3345-3353.

10. Kuecken, S.; Schmidt, J.; Zhi, L.; Thomas, A., Conversion of amorphous polymer networks to covalent organic frameworks under ionothermal conditions: A facile synthesis route for covalent triazine frameworks. Journal of Materials Chemistry A 2015, 3 (48), 24422-24427.

11. Soorholtz, M.; Jones, L. C.; Samuelis, D.; Weidenthaler, C.; White, R. J.; Titirici, M. M.; Cullen, D. A.; Zimmermann, T.; Antonietti, M.; Maier, J.; Palkovits, R.; Chmelka, B. F.; Schüth, F., Local Platinum Environments in a Solid Analogue of the Molecular Periana Catalyst. ACS Catalysis 2016, 6 (4), 2332-2340.

12. Gammon, W. J.; Kraft, O.; Reilly, A. C.; Holloway, B. C., Experimental comparison of N(1s) X-ray photoelectron spectroscopy binding energies of hard and elastic amorphous carbon nitride films with reference organic compounds. Carbon 2003, 41 (10), 1917-1923.

13. (a) Kapteijn, F.; Moulijn, J. A.; Matzner, S.; Boehm, H. P., Development of nitrogen functionality in model chars during gasification in $\mathrm{CO} 2$ and $\mathrm{O} 2$. Carbon 1999, 37 (7), 1143-1150; (b) Stańczyk, K.; Dziembaj, R.; Piwowarska, Z.; Witkowski, S., Transformation of nitrogen structures in carbonization of model compounds determined by XPS. Carbon 1995, 33 (10), 1383-1392.

14. (a) Arrigo, R.; Hävecker, M.; Schlögl, R.; Su, D. S., Dynamic surface rearrangement and thermal stability of nitrogen functional groups on carbon nanotubes. Chem. Commun. 2008, (40), 4891-4893; (b) Friedel Ortega, K.; Arrigo, R.; Frank, B.; Schlögl, R.; Trunschke, A., AcidBase Properties of N-Doped Carbon Nanotubes: A Combined Temperature-Programmed Desorption, X-ray Photoelectron Spectroscopy, and 2-Propanol Reaction Investigation. Chemistry of Materials 2016, 28 (19), 68266839. 
15. Beamson, G.; Briggs, D., High Resolution XPS of Organic Polymers: The Scienta ESCA300 Database. Wiley: 1992.

16. Tanuma, S.; Powell, C. J.; Penn, D. R., Calculations of electron inelastic mean free paths. V. Data for 14 organic compounds over the $50-2000 \mathrm{eV}$ range. Surface and Interface Analysis 1994, 21 (3), 165-176.
17. Smith, R. M.; Martell, A. E.; Standards, N. I. o.; Technology, NIST critically selected stability constants of metal complexes database. National Institute of Standards \& Technology: 1998. 